\title{
Small cycles in the Pancake graph
}

\author{
Elena Konstantinova * \\ Sobolev Institute of Mathematics, Novosibirsk, 630090, Russia \\ Department of Mathematics, Yeungnam University, 712-749, South Korea \\ Alexey Medvedev \\ Sobolev Institute of Mathematics, Novosibirsk, 630090, Russia \\ Central European University, Budapest, 1051, Hungary
}

Received 17 June 2011, accepted 23 April 2013, published online 26 April 2013

\begin{abstract}
The Pancake graph is well known because of the open Pancake problem. It has the structure that any $l$-cycle, $6 \leqslant l \leqslant n$ !, can be embedded in the Pancake graph $P_{n}, n \geqslant 3$. Recently it was shown that there are exactly $\frac{n !}{6}$ independent 6 -cycles and $n !(n-3)$ distinct 7 -cycles in the graph. In this paper we characterize all distinct 8-cycles by giving their canonical forms as products of generating elements. It is shown that there are exactly $\frac{n !\left(n^{3}+12 n^{2}-103 n+176\right)}{16}$ distinct 8-cycles in $P_{n}, n \geqslant 4$. A maximal set of independent 8cycles contains $\frac{n !}{8}$ of these.
\end{abstract}

Keywords: Cayley graphs, Pancake graph, cycle embedding, small cycles.

Math. Subj. Class.: 05C15, 05C25, 05C38, $90 B 10$

\section{Introduction}

The Pancake graph $P_{n}=\left(S y m_{n}, P R\right), n \geqslant 2$, is the Cayley graph on the symmetric group Sym $_{n}$ of permutations $\pi=\left[\pi_{1} \pi_{2} \ldots \pi_{n}\right]$, where $\pi_{i}=\pi(i)$ for any $1 \leqslant i \leqslant n$, with the generating set $P R=\left\{r_{i} \in S y m_{n}: 2 \leqslant i \leqslant n\right\}$ of all prefix-reversals $r_{i}$ reversing the order of any substring $[1, i], 2 \leqslant i \leqslant n$, of a permutation $\pi$ when multiplied on the right, i.e. $\left[\pi_{1} \ldots \pi_{i} \pi_{i+1} \ldots \pi_{n}\right] r_{i}=\left[\pi_{i} \ldots \pi_{1} \pi_{i+1} \ldots \pi_{n}\right]$. It is a connected vertex-transitive $(n-1)$ regular graph of order $n$ !. This graph is well known because of the combinatorial Pancake problem which was posed in [4] as the problem of finding its diameter. The problem is still

\footnotetext{
* The research was supported by grant 12-01-00448 of the Russian Foundation of Basic Research. The corresponding author.

E-mail addresses: e_konsta@math.nsc.ru (Elena Konstantinova), an_medvedev@yahoo.com (Alexey Medvedev)
} 
open. Some upper and lower bounds [5, 6] as well as exact values for $2 \leqslant n \leqslant 19$ [1, 2] are known. One of the main difficulties in solving this problem is the complicated cycle structure of the Pancake graph. This graph is hamiltonian [11] and the following result is also known.

Theorem 1.1. [7, 10] All cycles of length $l$, where $6 \leqslant l \leqslant n$ !, can be embedded in the Pancake graph $P_{n}, n \geqslant 3$, but there are no cycles of length 3,4 or 5 .

An explicit description of cycles is gradually being developed. The first results concerning cycle characterization in the Pancake graph were obtained in [8] where the following cycle representation via a product of generating elements was used. A sequence of prefixreversals $C_{l}=r_{i_{0}} \ldots r_{i_{l-1}}$, where $2 \leqslant i_{j} \leqslant n$, and $i_{j} \neq i_{j+1}$ for any $0 \leqslant j \leqslant l-1$, such that $\pi r_{i_{0}} \ldots r_{i_{l-1}}=\pi$, where $\pi \in S y m_{n}$, is called a form of a cycle $C_{l}$ of length $l$. A cycle $C_{l}$ of length $l$ is also called an $l$-cycle, and a vertex of $P_{n}$ is identified with the permutation which corresponds to this vertex. It is evident that any $l$-cycle can be presented by $2 l$ forms (not necessarily different) with respect to a vertex and a direction. The canonical form $C_{l}$ of an $l$-cycle is called a form with a lexicographically maximal sequence of indices $i_{0} \ldots i_{l-1}$. By using this description, the results characterizing 6 - and 7 -cycles were obtained in [8].

Theorem 1.2. [8] The Pancake graph $P_{n}, n \geqslant 3$, has $\frac{n !}{6}$ independent 6-cycles of the canonical form $C_{6}=r_{3} r_{2} r_{3} r_{2} r_{3} r_{2}$ and $n !(n-3)$ distinct 7 -cycles of the canonical form $C_{7}=r_{k} r_{k-1} r_{k} r_{k-1} r_{k-2} r_{k} r_{2}$, where $4 \leqslant k \leqslant n$. Each of the vertices of $P_{n}$ belongs to exactly one 6 -cycle and $7(n-3)$ distinct 7 -cycles.

The main result of this paper is the following theorem.

Theorem 1.3. Each of vertices of $P_{n}, n \geqslant 4$, belongs to $N=\frac{n^{3}+12 n^{2}-103 n+176}{2}$ distinct 8-cycles of the following canonical forms:

$$
\begin{array}{lr}
C_{8}^{1}=r_{k} r_{j} r_{i} r_{j} r_{k} r_{k-j+i} r_{i} r_{k-j+i}, & 2 \leqslant i<j \leqslant k-1,4 \leqslant k \leqslant n, \\
C_{8}^{2}=r_{k} r_{k-1} r_{2} r_{k-1} r_{k} r_{2} r_{3} r_{2}, & 4 \leqslant k \leqslant n, \\
C_{8}^{3}=r_{k} r_{k-i} r_{k-1} r_{i} r_{k} r_{k-i} r_{k-1} r_{i}, & 2 \leqslant i \leqslant k-2,4 \leqslant k \leqslant n, \\
C_{8}^{4}=r_{k} r_{k-i+1} r_{k} r_{i} r_{k} r_{k-i} r_{k-1} r_{i-1}, & 3 \leqslant i \leqslant k-2,5 \leqslant k \leqslant n, \\
C_{8}^{5}=r_{k} r_{k-1} r_{i-1} r_{k} r_{k-i+1} r_{k-i} r_{k} r_{i}, & 3 \leqslant i \leqslant k-2,5 \leqslant k \leqslant n, \\
C_{8}^{6}=r_{k} r_{k-1} r_{k} r_{k-i} r_{k-i-1} r_{k} r_{i} r_{i+1}, & 2 \leqslant i \leqslant k-3,5 \leqslant k \leqslant n, \\
C_{8}^{7}=r_{k} r_{k-j+1} r_{k} r_{i} r_{k} r_{k-j+1} r_{k} r_{i}, & 2 \leqslant i<j \leqslant k-1,4 \leqslant k \leqslant n, \\
C_{8}^{8}=r_{4} r_{3} r_{4} r_{3} r_{4} r_{3} r_{4} r_{3} . &
\end{array}
$$

There are two corollaries of Theorem 1.3, which will be proven in the final section of this paper.

Corollary 1.4. There are $\frac{n !\left(n^{3}+12 n^{2}-103 n+176\right)}{16}$ distinct 8 -cycles in $P_{n}, n \geqslant 4$.

Corollary 1.5. A maximal set of independent 8 -cycles in $P_{n}, n \geqslant 4$, contains $\frac{n !}{8}$ of these.

The proof of Theorem 1.3 is based on the hierarchical (recursive) structure of the Pancake graph which can be presented as follows. The graph $P_{n}, n \geqslant 3$, is constructed 
from $n$ copies of $P_{n-1}(i), 1 \leqslant i \leqslant n$, such that each $P_{n-1}(i)$ has the vertex set $V_{i}=$ $\left\{\left[\pi_{1} \ldots \pi_{n-1} i\right]\right.$, where $\left.\pi_{k} \in\{1, \ldots, n\} \backslash\{i\}: 1 \leqslant k \leqslant n-1\right\},\left|V_{i}\right|=(n-1) !$, and the edge set $E_{i}=\left\{\left\{\left[\pi_{1} \ldots \pi_{n-1} i\right],\left[\pi_{1} \ldots \pi_{n-1} i\right] r_{j}\right\}: 2 \leqslant j \leqslant n-1\right\},\left|E_{i}\right|=\frac{(n-1) !(n-2)}{2}$. Any two copies $P_{n-1}(i), P_{n-1}(j), i \neq j$, are connected by $(n-2)$ ! edges presented as $\left\{\left[i \pi_{2} \ldots \pi_{n-1} j\right],\left[j \pi_{n-1} \ldots \pi_{2} i\right]\right\}$, where $\left[i \pi_{2} \ldots \pi_{n-1} j\right] r_{n}=\left[j \pi_{n-1} \ldots \pi_{2} i\right]$. Prefixreversals $r_{j}, 2 \leqslant j \leqslant n-1$, define internal edges in all $n$ copies $P_{n-1}(i), 1 \leqslant i \leqslant n$, and the prefix-reversal $r_{n}$ defines external edges between copies. Copies $P_{n-1}(i)$, or just $P_{n-1}$ when it is not important to specify the last element of permutations belonging to the copy, are also called $(n-1)$-copies.

Since $P_{3} \cong C_{6}$ and due to the hierarchical structure, $P_{4}$ has four copies of $P_{3}$, each of which obviously cannot contain 8 -cycles. However, $P_{4}$ has 8 -cycles consisting of paths within copies of $P_{3}$ together with external edges between these copies. In general, any 8 -cycle of $P_{n}, n \geqslant 4$, must consist of paths within subgraphs that are isomorphic to $P_{k-1}$ for some $4 \leqslant k \leqslant n$, joined by external edges between these subgraphs. Hence, all 8-cycles of $P_{n}, n \geqslant 4$, could be found recursively by considering 8-cycles within each $P_{k}, 4 \leqslant k \leqslant n$, consisting of vertices from some copies of $P_{k-1}$. This approach is used in the proof of Theorem 1.3. To get the main result, we also need some technical lemmas concerning paths of length three between vertices of a given form. So, in the next section we introduce additional notations and prove two small lemmas. In Section 3 we prove Theorem 1.3 and its corollaries.

\section{Technical lemmas}

A segment $\left[\pi_{i} \ldots \pi_{j}\right]$ of a permutation $\pi=\left[\pi_{1} \ldots \pi_{i} \ldots \pi_{j} \ldots \pi_{n}\right]$ consists of all elements that lie between $\pi_{i}$ and $\pi_{j}$ inclusive. Any permutation can be written as a sequence of singleton and multiple segments. We use characters from $\{p, q, s, t\}$ to denote singletons and characters from $\{\alpha, \beta, \gamma, A, B, C\}$ to denote multiple segments. If $\pi=[\alpha \beta]$, where $\alpha=\left[\pi_{1} \pi_{2} \ldots \pi_{i}\right]$ and $\beta=\left[\pi_{i+1} \ldots \pi_{n}\right]$, then $\pi r_{|\alpha|}=[\bar{\alpha} \beta]$, where $|\alpha|$ is the number of elements in a segment $\alpha$, and $\bar{\alpha}$ is the inversion of a segment $\alpha$. It is obvious that $\overline{\bar{\alpha}}=\alpha$. Note that we allow empty segments where this does not contradict the initial definitions.

An independent set $D$ of vertices in a graph is called an efficient dominating set if each vertex not in $D$ is adjacent to exactly one vertex in $D$ [3]. It is known [9] that $D_{p}=$ $\left\{\left[p \pi_{2} \ldots \pi_{n}\right]: \pi_{j} \in\{1, \ldots, n\} \backslash\{p\}, 2 \leqslant j \leqslant n\right\},\left|D_{p}\right|=(n-1) !, 1 \leqslant p \leqslant n$, are efficient dominating sets in $P_{n}, n \geqslant 3$. Let us note that external edges of $P_{n}$ are incident to vertices from different efficient dominating sets of $P_{n}$. The distance $d=d(\pi, \tau)$ between two vertices $\pi, \tau$ in $P_{n}$ is defined as the least number of prefix-reversals transforming $\pi$ into $\tau$, i.e. $\pi r_{i_{1}} r_{i_{2}} \ldots r_{i_{d}}=\tau$.

The next lemma gives a full list of paths of length three between two vertices of the same efficient dominating set.

Lemma 2.1. Two permutations $\pi, \tau \in D_{p}, 1 \leqslant p \leqslant n$, are at distance three from each other in $P_{n}, n \geqslant 3$, if and only if:

1) either $\tau=\pi r_{j} r_{i} r_{j}, 2 \leqslant i<j \leqslant n$, where $\pi=[A B \gamma], \tau=[A \bar{B} \gamma]$;

2) or $\tau=\pi r_{j} r_{i} r_{i-j+1}, 2 \leqslant j<i \leqslant n$, where $\pi=[p A B \gamma], \tau=[p B A \gamma]$.

Proof. We consider $\pi \in D_{p}$ such that $\pi=[p \alpha q \beta k], \pi_{j}=q$. Let us find other vertices from $D_{p}$ being at the distance three from $\pi$. Let $\pi^{1}=\pi r_{j}=[q \bar{\alpha} p \beta k]$, where $\pi_{j}^{1}=p, 2 \leqslant$ $j \leqslant n$. An application of a prefix-reversal $r_{i}, 2 \leqslant i \leqslant n, i \neq j$, to the permutation $\pi^{1}$ gives us two cases: either $i<j$ or $i>j$. 
1) If $i<j$ then $\pi^{2}=\pi^{1} r_{i}=\left[\alpha_{2} q \overline{\alpha_{1}} p \beta k\right]$, where $\pi_{j}^{2}=p, \alpha=\alpha_{1} \alpha_{2}$ and $\left|\alpha_{2}\right|=i-1$, and then $\tau=\pi^{2} r_{j}=\left[p \alpha_{1} q \overline{\alpha_{2}} \beta k\right]$. Hence $\tau=\pi r_{j} r_{i} r_{j}$ and we get $\pi=[A B \gamma], \tau=[A \bar{B} \gamma]$ by setting $A=p \alpha_{1}, B=\alpha_{2} q, \gamma=\beta k$. Note, that using $r_{j}$ is the only way to restore $p$ to the first position and thus to end at an element of $D_{p}$ after reaching $\pi^{2}$.

2) If $i>j$ then $\pi^{2}=\pi^{1} r_{i}=\left[\overline{\beta_{1}} p \alpha q \beta_{2} k\right]$, where $\pi_{i-j+1}^{2}=p, \beta=\beta_{1} \beta_{2}$ and $\left|\beta_{1}\right|=i-j$, and then $\tau=\pi^{2} r_{i-j+1}=\left[p \beta_{1} \alpha q \beta_{2} k\right]$. Hence $\tau=\pi r_{j} r_{i} r_{i-j+1}$ and we get $\pi=[p A B \gamma], \tau=[p B A \gamma]$ by setting $A=\alpha q, B=\beta_{1}, \gamma=\beta_{2} k$. Note, that using $r_{i-j+1}$ is the only way to restore $p$ to the first position and thus to end at an element of $D_{p}$ after reaching $\pi^{2}$.

The next lemma gives a description of paths of length three defined on internal edges of the graph between vertices of given forms.

Lemma 2.2. Let permutations $\pi$ and $\tau$ be presented as:

1) $\pi=\left[\gamma_{1} A B \gamma_{2}\right]$ and $\tau=\left[\gamma_{1} \bar{A} B \gamma_{2}\right]$, where $\left|\gamma_{1}\right| \geqslant 1,|A| \geqslant 2$. Then:

a) there exists a unique path of length three:

$$
\tau=\pi r_{\left|\gamma_{1}\right|+|A|} r_{|A|} r_{\left|\gamma_{1}\right|+|A|},
$$

provided that either $\left|\gamma_{1}\right| \geqslant 2$ and $|A| \geqslant 2$, or $\left|\gamma_{1}\right|=1$ and $|A| \geqslant 3$;

b) there are two paths of length three:

$$
\tau=\pi r_{2} r_{3} r_{2}, \quad \tau=\pi r_{3} r_{2} r_{3}
$$

provided that $\left|\gamma_{1}\right|=1$ and $|A|=2$;

2) $\pi=\left[\gamma_{1} A B \gamma_{2}\right]$ and $\tau=\left[\gamma_{1} B A \gamma_{2}\right]$, where $\left|\gamma_{1}\right| \geqslant 0,|A| \geqslant 1,|B| \geqslant 1$. Then:

a) there is a unique path of length three:

$$
\tau=\pi r_{\left|\gamma_{1}\right|+2} r_{2} r_{\left|\gamma_{1}\right|+2},
$$

provided that $\left|\gamma_{1}\right| \geqslant 2$, and $|A|=|B|=1$;

$b)$ there is a unique path of length three:

$$
\tau=\pi r_{\left|\gamma_{1}\right|+|A|} r_{\left|\gamma_{1}\right|+|A|+|B|} r_{\left|\gamma_{1}\right|+|B|},
$$

provided that $\left|\gamma_{1}\right|=1$, and $|A| \neq 1$ or $|B| \neq 1$;

c) there are two paths of length three:

$$
\tau=\pi r_{2} r_{3} r_{2}=\pi r_{3} r_{2} r_{3},
$$

provided that $\left|\gamma_{1}\right|=|A|=|B|=1$;

d) there is a unique path of length three:

$$
\tau=\pi r_{|A|} r_{|A|+|B|} r_{|B|},
$$

provided that $\left|\gamma_{1}\right|=0$ and $|A| \geqslant 2,|B| \geqslant 2$.

There are no other paths of length three between $\pi$ and $\tau$ of these types. 
Proof. 1) If $\pi=\left[\gamma_{1} A B \gamma_{2}\right]$ and $\tau=\left[\gamma_{1} \bar{A} B \gamma_{2}\right]$, then (2.1) is checked by a direct verification: $\left[\gamma_{1} A B \gamma_{2}\right] \stackrel{r_{\left|\gamma_{1}\right|+|A|}}{\rightarrow}\left[\bar{A} \overline{\gamma_{1}} B \gamma_{2}\right] \stackrel{r_{|A|}}{\rightarrow}\left[A \overline{\gamma_{1}} B \gamma_{2}\right] \stackrel{r_{\left|\gamma_{1}\right|+|A|}}{\rightarrow}\left[\gamma_{1} \bar{A} B \gamma_{2}\right]$. Suppose that there is one more path of length three. Then these two paths should form a 6-cycle. In part (a), either $\left|\gamma_{1}\right| \geqslant 2$ and $|A| \geqslant 2$, or $\left|\gamma_{1}\right|=1$ and $|A| \geqslant 3$, so $r_{\left|\gamma_{1}\right|+|A|}=r_{m}$ for some $m \geqslant 4$, but by Theorem 1.2, no 6-cycle includes $r_{m}$ with $m \geqslant 4$ in its form. Therefore, the given path is the only one in this case. In part (b), $\left|\gamma_{1}\right|=1$ and $|A|=2$, so $m=3$ and the condition of Theorem 1.2 holds, hence we obtain two distinct paths of stated forms (2.2).

2) If $\pi=\left[\gamma_{1} A B \gamma_{2}\right]$ and $\tau=\left[\gamma_{1} B A \gamma_{2}\right]$, and $\left|\gamma_{1}\right| \geqslant 2,|A| \geqslant 1,|B| \geqslant 1$, then there is the following path of length four between these vertices:

$$
\begin{gathered}
\pi=\left[\gamma_{1} A B \gamma_{2}\right] \stackrel{r_{\left|\gamma_{1}\right|+|A|}}{\rightarrow}\left[\bar{A} \overline{\gamma_{1}} B \gamma_{2}\right] \stackrel{r_{\left|\gamma_{1}\right|+|A|+|B|}}{\rightarrow}\left[\bar{B} \gamma_{1} A \gamma_{2}\right] \stackrel{r_{\left|\gamma_{1}\right|+|B|}}{\rightarrow} \\
{\left[\overline{\gamma_{1}} B A \gamma_{2}\right] \stackrel{r_{\left|\gamma_{1}\right|}}{\rightarrow}\left[\gamma_{1} B A \gamma_{2}\right]=\tau .}
\end{gathered}
$$

Suppose there is also a path of length three between $\pi$ and $\tau$. By Theorem 1.1, there are no cycles of length 3 or 5 , and hence no paths of lengths 3 and 4 exist between two fixed vertices unless the paths are disjoint. This means that these two paths should form a 7-cycle, including the sequence $r_{m+a} r_{m+a+b} r_{m+b} r_{m}$, where $\left|\gamma_{1}\right|=m,|A|=a$ and $|B|=b$. By Theorem 1.2, this is possible only in the case when $m=k-2, k \geqslant 4$, and $a=b=1$, which implies that a unique path of length three has the form $r_{m+2} r_{2} r_{m+2}$ that corresponds to (2.3).

Putting $\left|\gamma_{1}\right|=1$ in (2.7), a path $\tau=\pi r_{\left|\gamma_{1}\right|+|A|} r_{\left|\gamma_{1}\right|+|A|+|B|} r_{\left|\gamma_{1}\right|+|B|}$, corresponding to (2.4), is obtained. Taking $\left|\gamma_{1}\right|=m,|A|=a$ and $|B|=b$, the obtained path is presented as $r_{m+a} r_{m+a+b} r_{m+b}$. Suppose that there is one more path of length three between $\pi$ and $\tau$. Then these two paths should form a 6 -cycle. By Theorem 1.2, this is possible only in the case when $m=a=b=1$, which gives us the paths $\tau=\pi r_{2} r_{3} r_{2}$ and $\tau=\pi r_{3} r_{2} r_{3}$, corresponding to (2.5).

Putting $\left|\gamma_{1}\right|=0$ in (2.7), a path $\tau=\pi r_{|A|} r_{|A|+|B|} r_{|B|}$, corresponding to (2.6) with $|A| \geqslant 2,|B| \geqslant 2$, is obtained. Suppose there is one more path of length three between $\pi$ and $\tau$. Then these two paths should form a 6-cycle. By the conditions of Lemma, $|A|+|B| \geqslant 4$, hence $r_{|A|+|B|}=r_{m}$ for some $m \geqslant 4$, but by Theorem 1.2, no 6-cycle includes $r_{m}$ with $m \geqslant 4$ in its form. Therefore, the given path is the only one in this case. If $|A|=1$ or $|B|=1$, then the path above is transformed into a 2-path or an edge. This completes the proof of the lemma.

\section{Proof of Theorem 1.3}

To find all 8-cycles passing through the same vertex in $P_{n}, n \geqslant 4$, we use its hierarchical structure by considering recursively 8 -cycles within each copy $P_{k}, 4 \leqslant k \leqslant n$, consisting of vertices from copies of $P_{k-1}$. It is assumed that any copy of $P_{k-1}$ has at least two vertices, since each vertex has a unique external edge. We obtain canonical forms of $8-$ cycles and count their numbers.

\section{Case 1: an 8-cycle within $P_{k}$ has vertices from two copies of $P_{k-1}$}

Suppose that an 8-cycle is formed on vertices from copies $P_{k-1}(p)$ and $P_{k-1}(s), 1 \leqslant$ $p \neq s \leqslant k$. It was shown in [8] that if two vertices $\pi$ and $\tau$, belonging the same $(k-1)-$ copy, are at the distance at most two, then their external neighbours $\bar{\pi}$ and $\bar{\tau}$ should belong to distinct $(k-1)$-copies. Hence, an 8-cycle cannot occur in situations when its two (three) 


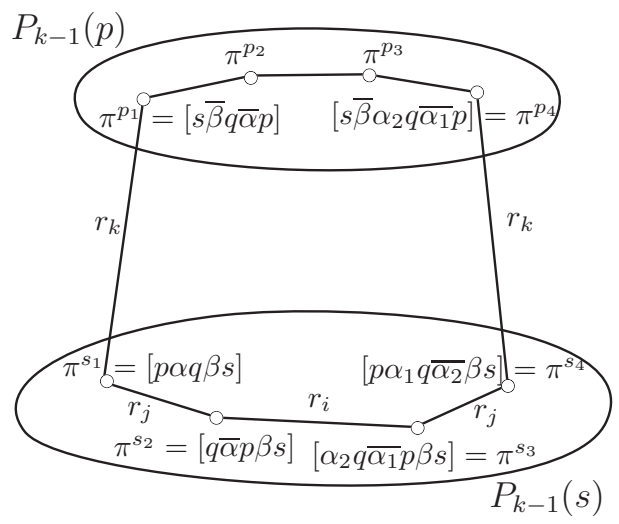

case 1

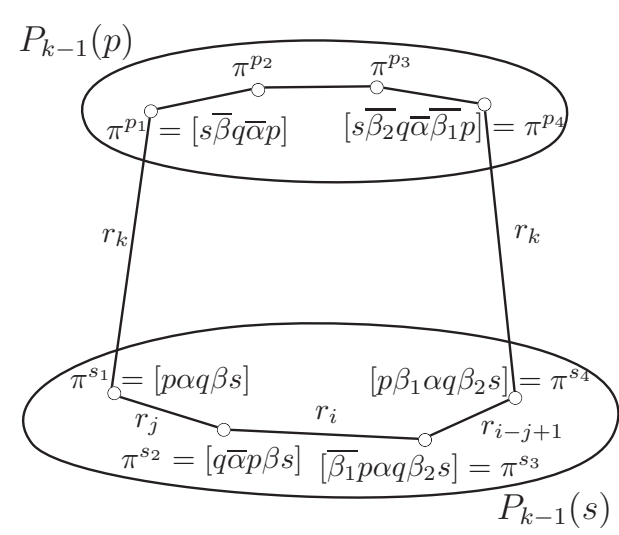

case 2

Figure 1: $(4+4)$-situation.

vertices belong to one copy and six (five) vertices belong to another one. Therefore, such an 8-cycle must have four vertices in each of the two copies.

$(4+4)$-situation. Suppose that four vertices of such an 8-cycle belong to a copy

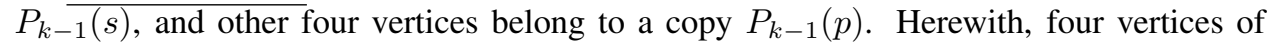
$P_{k-1}(s)$ should form a path of length three whose endpoints should be adjacent to vertices from $P_{k-1}(p)$, which means both vertices should belong to the efficient dominating set $D_{p}$. So, one vertex of $P_{k-1}(s)$ that is adjacent to a vertex of $P_{k-1}(p)$ must have the form $[p \alpha q \beta s]$. By Lemma 2.1, it is not hard to see that this gives rise to two possible forms for the remaining vertices of $P_{k-1}(s)$. These are given in Figure 1, where $|\alpha|=j-2$ and so $|\beta|=k-j-1$. In the first case we also have $\alpha=\alpha_{1} \alpha_{2}$ and $\left|\alpha_{2}\right|=i-1 \geqslant 1$, while in the second case we also have $\left|\beta_{1}\right|=i-j \geqslant 1$ and $\left|\beta_{2}\right|=k-i-1$.

Denote $\gamma_{1}=s \bar{\beta}, A=q \overline{\alpha_{2}}, B=\alpha_{1}, \gamma_{2}=p$, where $\left|\gamma_{1}\right|=|\beta|+1 \geqslant 1,|A| \geqslant 2$, $|B| \geqslant 0$, then in the first case $\pi^{p_{1}}, \pi^{p_{4}}$ have the forms $\left[\gamma_{1} A B \gamma_{2}\right]$ and $\left[\gamma_{1} \bar{A} B \gamma_{2}\right]$. By Lemma 2.2 (case 1a), there is a unique path of length three between these permutations if $\left|\gamma_{1}\right|=|\beta|+1=k-j \geqslant 1$ and $|A|=\left|\alpha_{2}\right|+1=i \geqslant 3$, or $k-j \geqslant 2$ and $i \geqslant 2$, and by Lemma 2.2 (case 1b), there are two distinct paths if $k-j=1$ and $i=2$. Hence, such an 8-cycle has the form $C_{8}^{1}=r_{k-j+i} r_{i} r_{k-j+i} r_{k} r_{j} r_{i} r_{j} r_{k}$, with $2 \leqslant i<j \leqslant k-1$, $4 \leqslant k \leqslant n$, the canonical form of which corresponds to (1.1). The case of $k-j=1$ and $i=2$ by symmetry gives one additional form $C_{8}^{2}=r_{k-1} r_{2} r_{k-1} r_{k} r_{2} r_{3} r_{2} r_{k}$, the canonical form of which corresponds to (1.2).

Denote $\gamma_{1}=s \overline{\beta_{2}}, A=\overline{\beta_{1}}, B=q \bar{\alpha}, \gamma_{2}=p$, where $\left|\gamma_{1}\right|=\left|\beta_{2}\right|+1 \geqslant 1,|A|=\left|\beta_{1}\right| \geqslant$ $1,|B|=|\alpha|+1 \geqslant 1$, then in the second case we have $\pi^{p_{1}}=\left[\gamma_{1} A B \gamma_{2}\right], \pi^{p_{4}}=\left[\gamma_{1} B A \gamma_{2}\right]$. By Lemma 2.2 (case 2a), there is a unique path of length three between $\pi^{p_{1}}$ and $\pi^{p_{4}}$ if $\left|\gamma_{1}\right|=k-i \geqslant 2,|A|=\left|\beta_{1}\right|=i-j=1$ and $|B|=|\alpha|+1=j-1=1$. Hence, $j=2$, $i=3$, and for $k \geqslant 5$ an 8-cycle has the form $r_{k-1} r_{2} r_{k-1} r_{k} r_{2} r_{3} r_{2} r_{k}$, corresponding again to the canonical form (1.2).

By Lemma 2.2 (case 2b), there also exists a unique path of length three between $\pi^{p_{1}}$ and $\pi^{p_{4}}$ if $\left|\gamma_{1}\right|=k-i=1,|A|=\left|\beta_{1}\right|=i-j \geqslant 1,|B|=|\alpha|+1=j-1 \geqslant 1$. This means that $i=k-1$, and such an 8-cycle has the form $C_{8}^{4}=r_{k} r_{k-j} r_{k-1} r_{j} r_{k} r_{k-j} r_{k-1} r_{j}$, $2 \leqslant j \leqslant k-2$, the canonical form of which corresponds to (1.3), if we set $j=i$. So, there 


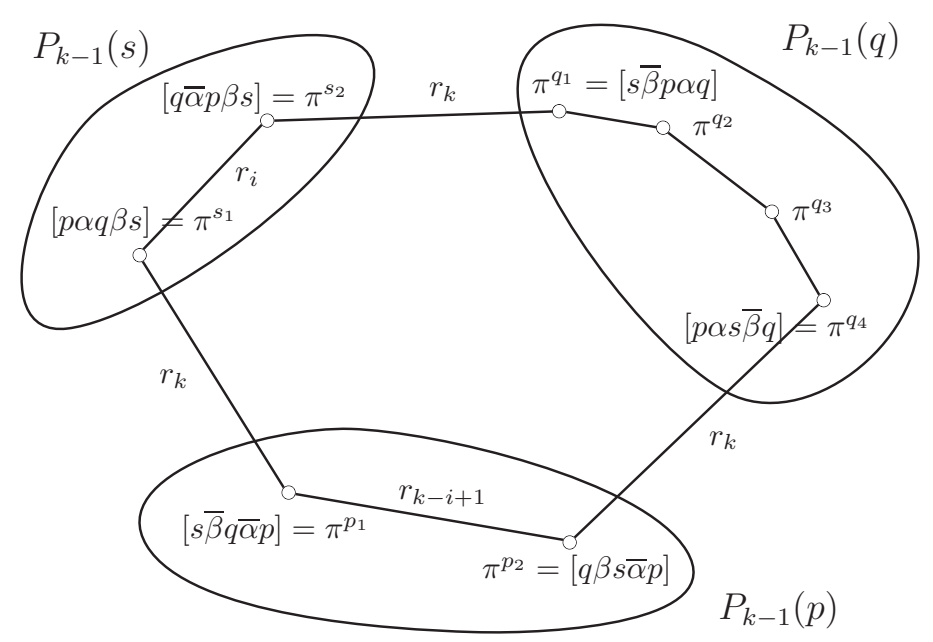

Figure 2: $(2+2+4)$-situation.

is a unique path of length three under the conditions listed, unless $|A|=|B|=1$ when by Lemma 2.2 (case 2c) this path is not unique. So, $k=4, j=2, i=3$ and 8-cycles take forms $r_{2} r_{3} r_{2} r_{4} r_{3} r_{2} r_{3} r_{4}$ and $r_{3} r_{2} r_{3} r_{4} r_{3} r_{2} r_{3} r_{4}$, corresponding to forms (1.2) and (1.1).

Thus, all 8-cycles occurring in the case of two copies are found.

\section{Case 2: an 8-cycle within $P_{k}$ has vertices from three copies of $P_{k-1}$}

Suppose an 8-cycle is formed on vertices from copies $P_{k-1}(p), P_{k-1}(q), P_{k-1}(s)$, where $1 \leqslant p \neq q \neq s \leqslant k$. There are following possible situations in this case.

$(2+2+4)$-situation. The distribution of vertices among the copies is presented by Figure 2. Let $\pi^{s_{1}}=[p \alpha q \beta s]$ where $\pi_{i}^{s_{1}}=q$ with $|\alpha|=i-2,|\beta|=k-i-1$. Then $\pi^{s_{2}}, \pi^{p_{1}}, \pi^{p_{2}}, \pi^{q_{1}}$ and $\pi^{q_{4}}$ are straightforward to define. Vertices $\pi^{q_{1}}$ and $\pi^{q_{4}}$ differ in the order of segments $s \bar{\beta}$ and $p \alpha$, hence they have the forms $\left[\gamma_{1} A B \gamma_{2}\right]$ and $\left[\gamma_{1} B A \gamma_{2}\right]$, where $\gamma_{1}$ is empty, $A=s \bar{\beta}, B=p \alpha, \gamma_{2}=q$ and $|A|=|\beta|+1 \geqslant 1,|B|=|\alpha|+1 \geqslant 1$. By Lemma 2.2 (case $2 \mathrm{~d}$ ), between $\pi^{q_{1}}$ and $\pi^{q_{4}}$ there exists a unique path of length three provided that $|A|=k-i \geqslant 2,|B|=i-1 \geqslant 2$, and no path of this length if $|A|=1$ or $|B|=1$. Thus, an 8-cycle has the form $C_{8}^{4}=r_{k} r_{k-i+1} r_{k} r_{i-1} r_{k-1} r_{k-i} r_{k} r_{i}$, where $3 \leqslant i \leqslant k-2, k \geqslant 5$, the canonical form of which corresponds to (1.4).

$(2+3+3)$-situation. The distribution of vertices among the copies is presented by Figure 3. Let $\pi^{s_{1}}=[p \alpha q \beta s]$, where $|\alpha|=i-2$ and $|\beta|=k-i-1$. Then $\pi^{s_{2}}, \pi^{p_{1}}$ and $\pi^{q_{1}}$ are straightforward to define. Since $\pi^{p_{3}}$ and $\pi^{q_{3}}$ are joined by an external edge, $\pi_{1}^{p_{3}}=q$. Moreover, $\pi^{p_{1}}$ and $\pi^{p_{3}}$ should be joined by a path of length two that can be obtained by two ways:

$$
\pi^{p_{1}}=[s \bar{\beta} q \bar{\alpha} p] \rightarrow \begin{cases}{\left[\beta_{2} s \overline{\beta_{1}} q \bar{\alpha} p\right] \rightarrow\left[q \beta_{1} s \overline{\beta_{2}} \bar{\alpha} p\right]=\pi^{p_{3}^{1}},} & \text { where }\left|\beta_{2}\right| \neq 0 \\ {\left[\alpha_{2} q \beta s \overline{\alpha_{1}} p\right] \rightarrow\left[q \overline{\alpha_{2}} \beta s \overline{\alpha_{1}} p\right]=\pi^{p_{3}^{2}},} & \text { where }\left|\alpha_{2}\right| \neq 0 .\end{cases}
$$

From the other side, $\pi^{q_{3}}$ and $\pi^{p_{3}}$ are joined by an external edge, hence $\pi_{1}^{q_{3}}=p$, and there 


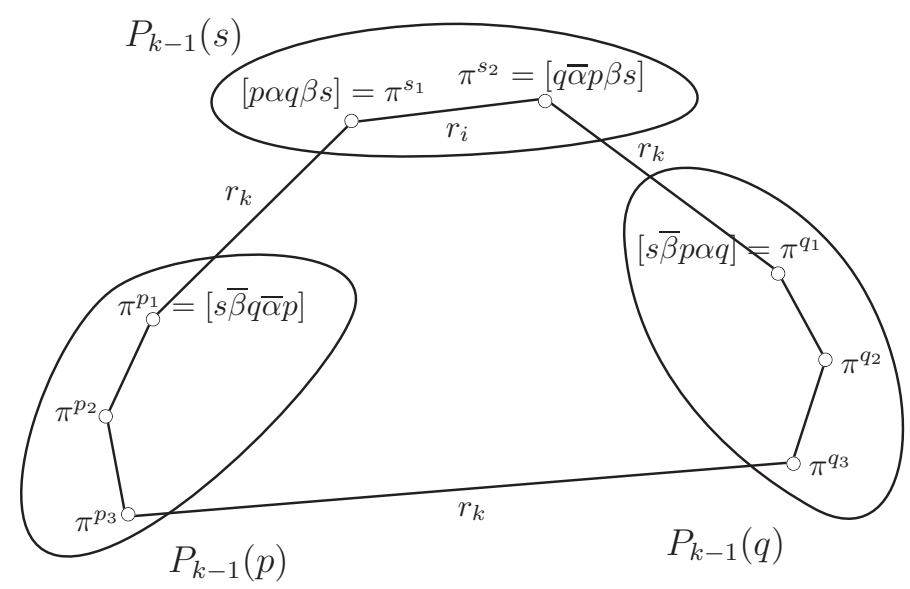

Figure 3: $(2+3+3)$-situation.

should be a path of length two between $\pi^{q_{1}}$ and $\pi^{q_{3}}$ such that:

$$
\pi^{q_{1}}=[s \bar{\beta} p \alpha q] \rightarrow \begin{cases}{\left[\beta_{2} s \overline{\beta_{1}} p \alpha q\right] \rightarrow\left[p \beta_{1} s \overline{\beta_{2}} \alpha q\right]=\pi^{q_{3}^{1}},} & \text { where }\left|\beta_{2}\right| \neq 0 . \\ {\left[\overline{\alpha_{1}} p \beta s \alpha_{2} q\right] \rightarrow\left[p \alpha_{1} \beta s \alpha_{2} q\right]=\pi^{q_{3}^{2}},} & \text { where }\left|\alpha_{1}\right| \neq 0 .\end{cases}
$$

Analysis of non-empty segments in these permutations shows that external edges occur between: $\pi^{p_{3}^{1}}$ and $\pi^{q_{3}^{2}}$, if $\left|\alpha_{2}\right|=0,\left|\beta_{1}\right|=0 ; \pi^{p_{3}^{2}}$ and $\pi^{q_{3}^{1}}$, if $\left|\alpha_{1}\right|=0,\left|\beta_{1}\right|=0 ; \pi^{p_{3}^{2}}$ and $\pi^{q_{3}^{2}}$, if $|\beta|=0$. There is no external edge between $\pi^{p_{3}^{1}}$ and $\pi^{q_{3}^{1}}$ since they have the same order of elements in the segment $s \overline{\beta_{2}}$.

Since $|\alpha|=i-2,|\beta|=k-i-1$, then using the edge between $\pi^{p_{3}^{1}}$ and $\pi^{q_{3}^{2}}$, where $\left|\alpha_{2}\right|=0,\left|\beta_{1}\right|=0$, we have $|\alpha| \geqslant 1,|\beta| \geqslant 1$, and such an 8-cycle has the form $C_{8}^{5}=r_{k} r_{k-i} r_{k-i+1} r_{k} r_{i-1} r_{k-1} r_{k} r_{i}$, with $3 \leqslant i \leqslant k-2,5 \leqslant k \leqslant n$, the canonical form of which corresponds to (1.5). Using the external edge between $\pi^{p_{3}^{2}}$ and $\pi^{q_{3}^{1}}$, where $\left|\alpha_{1}\right|=0,\left|\beta_{1}\right|=0$, we have $|\alpha| \geqslant 1,|\beta| \geqslant 1$, and such an 8-cycle has the form $r_{k} r_{k-1} r_{i-1} r_{k} r_{k-i+1} r_{k-i} r_{k} r_{i}$, where $3 \leqslant i \leqslant k-2$, the canonical form of which also corresponds to the form (1.5). Finally, using the external edge between $\pi^{p_{3}^{2}}$ and $\pi^{q_{3}^{2}}$, where $|\beta|=0$, we have $i=k-1,\left|\alpha_{1}\right|=j \geqslant 1,\left|\alpha_{2}\right|=k-3-j \geqslant 1$, so there is one more 8-cycle of the form $C_{8}^{6}=r_{k} r_{k-j-1} r_{k-j-2} r_{k} r_{j+1} r_{j+2} r_{k} r_{k-1}$, where $1 \leqslant j \leqslant k-4$, $5 \leqslant k \leqslant n$, the canonical form of which corresponds to (1.6), if we put $j=i-1$.

Thus, all 8-cycles occurring in the case of three copies are found.

\section{Case 3: an 8-cycle within $P_{k}$ has vertices from four copies of $P_{k-1}$}

The distribution of vertices among the copies is presented by Figure 4. Let $\pi^{q_{1}}=$ $[s \alpha t \beta p \gamma q]$, where $|\alpha| \geqslant 0,|\beta| \geqslant 0,|\gamma| \geqslant 0$. There are two cases.

1) Suppose that $\pi^{q_{1}}$ is adjacent to $\pi^{s_{1}}$, and $\pi^{q_{2}}$ is adjacent to $\pi^{t_{1}}$. Since there is only one cycle edge within each copy, hence this edge is uniquely defined and all vertices' labels are straightforward to obtain (see Figure 4, case 1). Thus, we end up with $\pi^{p_{1}}=[s \alpha t \beta q \bar{\gamma} p]$, $\pi^{p_{2}}=[t \bar{\alpha} s \beta q \bar{\gamma} p]$. If an 8 -cycle does exist, then $\pi^{p_{1}}, \pi^{p_{2}}$ should be incident to the same 

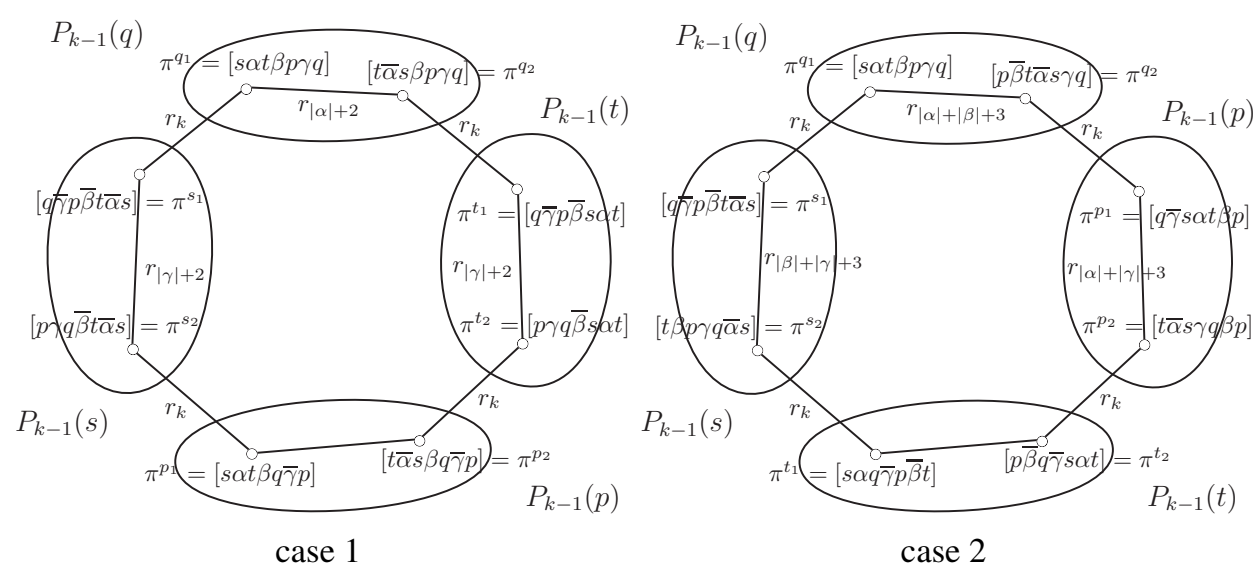

Figure 4: $(2+2+2+2)$-situation.

internal edge, and hence, there should exist a prefix-reversal transforming $\pi^{p_{1}}$ into $\pi^{p_{2}}$, namely, $r_{|\alpha|+2}$. If we set $|\alpha|=i-2,|\beta|=j-i-1,|\gamma|=k-j-1$, where $2 \leqslant i<j<k$, then such an $8-$ cycle is presented by the form $C_{8}^{7}=r_{k} r_{k-j+1} r_{k} r_{i} r_{k} r_{k-j+1} r_{k} r_{i}$, where $2 \leqslant i<j \leqslant k-1,4 \leqslant k \leqslant n$, the canonical form of which corresponds to (1.7).

2) Suppose that $\pi^{q_{1}}$ is adjacent to $\pi^{s_{1}}$, and $\pi^{q_{2}}$ is adjacent to $\pi^{p_{1}}$ (see Figure 4, case $2)$, then we end up with $\pi^{t_{1}}=[s \alpha q \bar{\gamma} p \bar{\beta} t], \pi^{t_{2}}=[p \bar{\beta} q \bar{\gamma} s \alpha t]$. In this case, an internal edge between vertices $\pi^{t_{1}}$ and $\pi^{t_{2}}$ does exist only if $|\alpha|=|\beta|=|\gamma|=0$, which means that $k=4$ and such an 8-cycle takes the form (1.8).

Therefore, all canonical forms for 8-cycles in $P_{n}, n \geqslant 4$, are obtained.

Now we count the total number $N=\sum_{i=1}^{8} N_{C_{8}^{i}}$ of distinct 8-cycles passing through a given vertex, where $N_{C_{8}^{i}}$ corresponds to the number of distinct 8-cycles described by the canonical form $C_{8}^{i}, 1 \leqslant i \leqslant 8$. Let us note that any canonical form of an $l$-cycle describes $l$ cycles (not necessarily distinct) for a given vertex. Among all canonical forms (1.1) (1.8), there is the only one, namely the form (1.5), which describes eight distinct 8cycles. In other cases, identical forms occur. For example, from the canonical form $C_{8}^{8}=$ $r_{4} r_{3} r_{4} r_{3} r_{4} r_{3} r_{4} r_{3}$ one can get two forms, namely, $r_{4} r_{3} r_{4} r_{3} r_{4} r_{3} r_{4} r_{3}$ and $r_{3} r_{4} r_{3} r_{4} r_{3} r_{4} r_{3} r_{4}$ which are identical because they describe the same 8 -cycle. Thus, the canonical form $C_{8}^{8}$ gives the only 8-cycle, hence, $N_{C_{8}^{8}}=1$. In other cases, it can be shown in the same manner (by taking into account identical forms) that the numbers $N_{C_{8}^{i}}, 1 \leqslant i \leqslant 7$, are given as follows: $N_{C_{8}^{1}}=\frac{(n-3)(n-2)(n-1)}{3}, N_{C_{8}^{2}}=4(n-3), N_{C_{8}^{3}}=(n-2)(n-3)$, $N_{C_{8}^{4}}=N_{C_{8}^{6}}=2(n-3)(n-4), N_{C_{8}^{5}}=4(n-3)(n-4), N_{C_{8}^{7}}=\frac{(n-3)(n-2)(n-1)}{6}$. Thus, the total number is given by

$$
N=\frac{n^{3}+12 n^{2}-103 n+176}{2},
$$

which completes the proof of the theorem.

The total number of distinct 8 -cycles in $P_{n}, n \geqslant 4$, is given by $\frac{n !\left(n^{3}+12 n^{2}-103 n+176\right)}{16}$ since there are $n$ ! vertices in the graph each of which belongs to $N$ distinct 8 -cycles. This proves Corollary 1.4. 
A maximal set of independent $8-$ cycles in $P_{n}, n \geqslant 4$, contains $\frac{n !}{8}$ of these, since $P_{4}$ has three independent 8 -cycles, and there are $\frac{n !}{24}$ copies of $P_{4}$, each of which consists of exactly three independent $8-$ cycles. This proves Corollary 1.5.

\section{Acknowledgment}

The authors thank the referees for their critical remarks and valuable suggestions, which helped to improve the quality and clarity of the manuscript.

\section{References}

[1] S. Asai, Y. Kounoike, Y. Shinano and K. Kaneko, Computing the diameter of 17-pancake graph using a PC cluster, LNCS 4128 (2006), 1114-1124.

[2] J. Cibulka, On average and highest number of flips in pancake sorting, Theoretical Computer Science 412 (2011), 822-834.

[3] I. J. Dejter, O. Serra, Efficient dominating sets in Cayley graphs, Discrete Appl. Math. 129 (2003), 319-328.

[4] H. Dweighter, E 2569 in: Elementary problems and solutions, Amer. Math. Monthly 82 (1975) 1010.

[5] W. H. Gates and C. H. Papadimitriou, Bounds for sorting by prefix-reversal, Discrete Math. 27 (1979), 47-57.

[6] M. H. Hyedari and I. H. Sudborough, On the diameter of the pancake network, Journal of Algorithms 25 (1997), 67-94.

[7] A. Kanevsky and C. Feng, On the embedding of cycles in pancake graphs, Parallel Computing 21 (1995), 923-936.

[8] E. V. Konstantinova and A. N. Medvedev, Cycles of length seven in the Pancake graph, Diskretn. Anal. Issled. Oper. 17 (2010), 46-55 (in Russian).

[9] Ke Qiu, Optimal broadcasting algorithms for multiple messages on the star and pancake graphs using minimum dominating sets, Congress. Numerantium. 181 (2006), 33-39.

[10] J. J. Sheu, J. J. M. Tan and K. T. Chu, Cycle embedding in pancake interconnection networks, Proc. 23rd Workshop on Combinatorial Mathematics and Computation Theory, Taiwan (2006), pp. 85-92.

[11] S. Zaks, A new algorithm for generation of permutations, BIT 24 (1984), 196-204. 